\title{
Mondialisation, disjonctions, émiettements culturels, terrain : les enjeux du militantisme ethnologique
}

Jean-Pierre Warnier

\section{OpenEdition}

\section{Journals}

Édition électronique

URL : https://journals.openedition.org/pa/1838

DOI : 10.4000/pa.1838

ISSN : 2273-0362

Éditeur

Université Lumière Lyon 2

\section{Édition imprimée}

Date de publication : 1 janvier 2005

Pagination : 5-9

ISBN : 1634-7706

ISSN : 1634-7706

Référence électronique

Jean-Pierre Warnier, « Mondialisation, disjonctions, émiettements culturels, terrain : les enjeux du militantisme ethnologique », Parcours anthropologiques [En ligne], 5 | 2005, mis en ligne le 15 juillet 2021, consulté le 21 juillet 2021. URL : http://journals.openedition.org/pa/1838 ; DOl : https://doi.org/ $10.4000 /$ pa. 1838 


\section{«MONDialisation, DisJonCtions, ÉMIETTEMENTS CULTURELS, TERRAIN : LES ENJEUX DU MILITANTISME ETHNOLOGIQUE "}

En 1999, je publiai un petit ouvrage intitulé $L a$ mondialisation de la culture (La Découverte, coll. "Repères ", $3^{\text {ème }}$ édition 2004). En toute immodestie, son succès fut immédiat et ne s'est pas démenti depuis. Il en est à sa troisième édition. Ce livre était une commande de la maison d'édition. J'avoue qu'il se situe à la périphérie de mes préoccupations, mais son destin m'a enseigné beaucoup de choses. Sur le titre d'abord : il ne me plaisait guère car je soutenais que la thèse d'une mondialisation de la culture (disons pour faire bref d'une américanisation) ne résiste pas à l'examen. Je suggérai à l'éditeur de rajouter au moins un point d'interrogation, voire même de titrer La non mondialisation de la culture. Pour des raisons éditoriales et commerciales, il refusa. Je pense en fin de compte qu'il avait raison. Ensuite, je me suis rendu compte que ce livre modeste (en termes d'investissement, de travail et d'ambitions) se vend comme des petits pains alors que j'essaie en vain depuis trois ans de publier l'œuvre de quinze ans, à laquelle je tiens beaucoup... Ainsi va le travail de l'intellectuel.

Ces anecdotes illustrent une difficulté que j'ai éprouvée depuis la sortie de la première édition de La mondialisation de la culture. Un livre de ce genre exige une sorte de service après vente sous forme de conférences, interventions à la radio (France Culture, RFI, Radio Aligre) entretiens publiés dans des magazines, tables rondes, etc. De sorte que six ans après sa sortie j'ai acquis une certaine expérience de sa réception et du débat français sur la «non mondialisation" de la culture et sur la "diversité culturelle" (anciennement « exception culturelle »).

Une chose m'apparaît: il m'est pratiquement impossible de situer le débat là où les vraies questions me semblent se poser, encore moins sur les sujets qui me semblent importants, comme celui dont traite le livre que j'essaie en vain de publier. Pour la grande majorité des journalistes et du public, la question centrale est la suivante : le métissage culturel (cf. Laplantine et Nouss, 1997), qui est une évidence universellement partagée, nous conduit-il vers une culture planétaire unique (entendez américaine) par convergence des civilisations? Allons-nous perdre notre âme, nous fondre dans un grand tout indifférencié ? Autrement dit, le sens commun ne semble pas avoir bougé depuis les années 1960 lorsque la théorie de la modernisation s'en tenait à la " convergence " de toutes les civilisations vers un modèle unique, industriel, démocratique, inspiré des Lumières, bref, occidental. La lecture vulgaire du Choc des civilisations de Huntington (1996) n'a rien fait pour clarifier les termes du débat. Si bien qu'intervention après intervention, il faut rappeler : "non, il n'y a pas mondialisation de la culture, non le métissage n'entraîne pas uniformisation, le vrai débat est ailleurs, il est politique. C'est une question de régulation des conflits et de mobilisation des cultures, etc. »

\section{La posture ethnologique}

Dans le livre et dans mes interventions, je m'efforce de décaler le débat dans trois directions :

$1 \%$ La plupart des débats sont cadrés par un présupposé implicite qui est le suivant : la culture serait une entité autonome soumise à ses propres dynamiques. La culture américaine, par exemple, se diffuserait par contagion, par imprégnation. En solution dans le Coca Cola, elle se répandrait dans le monde du seul fait que les consommateurs boiraient le breuvage emblématique des États Unis. Contre ce point de vue simpliste, je ne manque pas une occasion de rappeler qu'il n'y a pas de culture qui ne soit celle d'une société ou d'un groupe donné, et pas de société ou de groupe qui ne possède une culture. En conséquence les dynamiques culturelles sont articulées aux dynamiques sociopolitiques des groupes. Leur autonomie est toute relative. Il faut en faire une lecture politique. Étant donné que l'humanité est une machine à produire des conflits, du quant à soi, de la divergence d'intérêts et de points de vue, ainsi que des alliances, c'est également une machine à produire de la différence culturelle, de l'identité, y compris par des métissages sélectifs. En conséquence, la perspective d'une uniformisation culturelle de la planète ou d'une "mondialisation de la culture" est aussi improbable que la paix universelle. La thèse d'une uniformisation ne résiste pas à l'examen, ne serait-ce que pour cette seule raison. En outre, il est contre-productif de poser cette question, parce qu'elle occulte des débats plus radicaux, d'ordre anthropologique et politique. Malgré les prises de position très argumentées de J.-F. Bayart (1996, 2004), de J.-L. Amselle (2001), et de plusieurs autres, le débat a du mal à sortir de l'ornière du culturalisme spontané et naïf.

$2 \%$ Les risques de fragmentation et de balkanisation culturelle semblent en effet beaucoup plus préoccupants. Mais ils sont mal ou à peine perçus. Cette question a été soulevée par Norbert Elias de manière quasi lancinante depuis 1945 (en particulier dans Qu'est-ce que la sociologie? 1970/1991). Elias constate l'intensification des interactions entre tous les groupes sociaux, nations, États, etc. alors même que chacun de ces groupes continue d'entretenir une perception locale, partielle, limitée, de ces interactions. La connaissance des interrelations reste à la traîne, et l'action collective des groupes locaux reste tragiquement inadéquate à l'extension régionale, continentale ou mondiale des interactions. Les exemples sont si nombreux 
qu'on hésite à en citer ne serait-ce qu'un ou deux à titre d'illustration.

Il le faut bien cependant, afin de ne pas se perdre dans les généralités. Exemple : les chasseurs de la baie de Somme et du littoral occidental de la France en général défendent leurs pratiques cynégétiques alors que les migrateurs qu'ils chassent parcourent toute l'Europe. Depuis la fin des années 1990, en vue de différentes élections, ils constituèrent un mouvement "Chasse, pêche, nature, traditions " explicitement anti-européen alors que la question environnementale est devenue européenne et même mondiale. On m'objectera qu'ils en sont bien conscients mais qu'ils contestent que la Commission de Bruxelles soit qualifiée pour traiter d'un problème supranational. Ils estiment qu'il faut le résoudre dans le cadre national. C'est bien là que l'argument de N. Elias me semble pertinent: j'estime que ces électeurs sont d'une grande ignorance au regard de tout ce qui se passe audelà des limites de leur canton, et ceci à tous égards: historique, politique, culturel, environnemental, cynégétique. Il faut le dire : ils sont ignares. Quitte à encourir les foudres de la corporation, j'oserai dire que la communauté des enseignants $\mathrm{du}$ supérieur en France nous donnerait de nombreux exemples du même tonneau. J'ai pu constater à maintes reprises l'ignorance et le désintérêt de la grande majorité d'entre eux par rapport aux dynamiques européennes et mondiales. Cela ne veut pas dire que nombreux sont celles et ceux (comme les ethnologues) qui sont fort compétents sur un canton exotique du vaste monde, mais cela n'entraîne pas ipso facto une perception globale $\mathrm{du}$ contexte universitaire européen qui les sensibilise à l'importance de programmes d'action qui débordent les frontières de l'hexagone.

$3 \%$ C'est en fait à l'ethnologie que je veux en venir, car je suis convaincu que, dans le concert des sciences de l'homme et de la société, l'ethnologie occupe une place stratégique et originale. C'est toujours en me réclamant de mon métier d'ethnologue, témoin du rapport entre le local et le global, que je développe mes arguments sur la non mondialisation de la culture. La question ultime que je me pose est donc celle du rôle que joue aujourd'hui l'ethnologie dans ce débat public. Ce rôle, on ne peut l'évaluer qu'en diachronie par rapport à celui qu'elle a joué en France et ailleurs, depuis la deuxième guerre mondiale. Mon ambition n'est pas d'esquisser les grandes lignes de l'histoire publique de la discipline. L'affaire est trop complexe pour être abordée dans un court article. Je vais me concentrer sur une seule question : il me semble qu'on peut dire, sans trop de risques de se tromper, que les ethnologues français d'obédiences très diverses, qui ont percé auprès $\mathrm{du}$ grand public (Leroi-Gourhan, Leiris, Rouch, Godelier, Augé, et surtout Lévi-Strauss) ont été les témoins efficaces de la diversité culturelle, y compris du proche. Même si le néo-colonialisme n'est pas mort dans la pratique, ses bases idéologiques ont été déplacées. L'argumentaire colonial revient souvent à la charge, mais il convainc de moins en moins.

Les noms que je cite ne doivent pas occulter l'importance déterminante de collègues qui n'ont pas forcément bénéficié des feux de la rampe, mais qui, par leurs compétences, leur nombre croissant à partir des années 1950, ont labouré l'ensemble du champ, et touché les publics les plus divers.

Le succès de l'entreprise est attesté par la fortune du mot "culture", et pas son enrichissement sémantique grâce à l'apport de l'ethnologie (on en a de multiples indicateurs: de la référence explicite faite par l'École des Annales à l'ethnologie, au contenu des manuels des classes de première et de terminale SES qui citent copieusement les ethnologues). Mais le mot de "culture ", son contenu et ses enjeux se disputent entre les ethnologues d'une part et les spécialistes des industries de la culture ou des "pratiques culturelles " d'autre part, autrement dit, des spécialistes de "l'info-com». Cela provoque un quiproquo sur lequel je reviendrai ci-dessous. En attendant, je me pose la question suivante: pourquoi l'ethnologie échoue-t-elle aujourd'hui à déstabiliser le sens commun sur la question de la convergence ou de la mondialisation de la culture, et à placer le débat sur les rapports entre le local et le global, ou, en termes éliasiens, entre les groupes locaux et les réseaux d'interactions de différents niveaux hiérarchiques ? Pourquoi s'avère-t-il si difficile de faire entendre d'autres débats que celui d'une uniformisation imaginaire?

\section{Les ethnologues sont-ils passés à côté du débat?}

Après tout, le débat sur la convergence des civilisations a déjà 150 ans d'existence puisque ses termes ont été clairement posés par Gobineau en 1859. Dans sa conclusion générale, il filait la métaphore d'une histoire humaine "semblable à une toile immense ", faite de mille matériaux et de mille couleurs, mais que les mélanges semblent devoir inéluctablement réduire à une morne grisaille. Et d'ajouter, je cite :

"Mélange, mélange partout, toujours mélange, voilà l'œuvre la plus claire, la plus assurée, la plus durable des grandes sociétés et des puissantes civilisations, celle qui, à coup sûr, leur survit; et plus les premières ont d'étendue territoriale et les secondes de génie conquérant, plus loin les flots ethniques qu'elles soulèvent vont saisir d'autres flots primitivement étrangers, ce dont leur nature et la sienne s'altèrent également »(Gobineau, 1853/1983 : $1159-1160)^{1}$.

Ce point de vue sur «l'altération» des civilisations par leur mélange et leur uniformisation a été souvent et doctement repris et abondé par les plus grands (y compris Mauss, et Lévi-Strauss qui professait une grande admiration pour l'œuvre de Gobineau et en faisait un pré-structuraliste). Il ne faut pas s'étonner qu'il ait la vie dure. Je me suis donc demandé si les ethnologues avaient tardé à réagir ou avaient échoué à poser clairement la question du rapport entre le local et le global.

N'étant pas spécialiste de l'histoire de l'anthropologie, je n'ai pas de certitude. Mais je dispose d'un jalon solide : en 1993 s'est tenue à Oxford la quatrième "Decennial Conference " de la «Anthropological Studies Association" à laquelle j'ai eu la chance de participer ${ }^{2}$. Le thème en était le suivant: "The uses of knowledge. Global and local relations » («L'usage du savoir. 
Les relations global-local »). Ce thème avait été choisi dès 1989 sur la base de travaux accomplis depuis le début des années 1980. Rappelons qu'on s'accorde à dater du milieu des années 1970 la montée en puissance des flux mondialisés de capitaux, de marchandises et de contenus médiatiques, sous l'impact des chocs pétroliers et des différents cycles du GATT. Ces événements ont relancé les spéculations sur la convergence des cultures par mondialisation des flux culturels.

Je ne sais pas si la "ASA Conference" de 1993 eut son équivalent du côté francophone. En tout état de cause, la preuve par ASA est formelle : il était évident, pour les 450 participants du colloque (dont 3 français ; dont deux appartenant à des universités britanniques) que le débat sur la convergence était une affaire classée sur laquelle il était inutile de revenir. Il était clair qu'on ne constatait ni convergence des civilisations, ni mondialisation de la culture. Il était tout aussi clair que le vrai débat pouvait être avantageusement posé en termes de "savoirs" ou de «intelligence» (entendez acquisition de savoir sur les autres sociétés ou groupes, ou encore « renseignement»).

Très en gros, la question posée était la suivante : étant entendu que la culture n'est pas réductible au savoir, quels sont les savoirs, les renseignements dont disposent les sujets dans les relations local-local et local-global et qui sont un facteur clé des relations interculturelles? Il s'agit du savoir que possèdent les sujets sur eux-mêmes, sur les groupes auxquels ils appartiennent, sur les autres groupes et sur les flux mondialisés.

En 1990, Arjun Appadurai, dans un article qui fait date, avait noté l'existence de "disjonctions " entre ces savoirs et, au bout du compte, entre les États et tout ce qui est localement enraciné, qui fait identité et qui relève de ce qu'on appelle habituellement la "nation". S'inspirant de B. Anderson et de ses analyses des " communautés imaginées ", A. Appadurai notait que les sujets localisés (par opposition aux sujets « globalisés " par leur travail, leur résidence, leur mode de vie et l'organisation à laquelle ils appartiennent) ne disposent d'aucun savoir adéquat sur les flux financiers, marchands et médiatiques globalisés, à l'impact desquels ils sont quotidiennement exposés. Il en résulte que les sujets localisés produisent des savoirs de substitution, largement imaginaires, disjoints des réalités globales, et qu'A. Appadurai désigne par les néologismes de "financescape, mediascapes, etc. " (paysages financiers, médiatiques, etc.).

A. Appadurai n'avait pas pu participer au colloque ASA, mais il avait envoyé une contribution intitulée "The production of locality" reprise plus tard dans son livre Modernity at large (1997) si médiocrement traduit en français qu'il n'a guère trouvé d'écho de ce côté-ci de l'Atlantique. Pire : cette traduction a discrédité une réflexion dont la pertinence me semble évidente.

Le colloque ASA s'est efforcé d'accomplir un repérage de la diversité des savoirs qu'ont les groupes sociaux à l'égard les uns des autres par de là les frontières socioculturelles. Il s'est ensuite efforcé de saisir cette diversité dans des domaines où l'on peut constater la circulation d'items culturels d'un groupe à l'autre : les images, les pratiques religieuses, les marchandises, le management d'entreprise, les technologies de la reproduction humaine, la consommation, la sexualité, le genre, ... et aussi la connaissance que les différentes communautés d'anthropologues possèdent les unes sur les autres (vaste programme !).

La conclusion de ce colloque, c'est que les anthropologues de langue anglaise, comme les Français, ont décroché du débat sur la convergence dès les années 1980, que, pour eux, comme pour les ethnologues français, la diversité culturelle est un fait durable et avéré, et que "en théorie ", il n'existe aucune raison de penser qu'elle puisse céder à une uniformisation dans la mesure où le « métissage » est mobilisé au service de la production de la différence comme de l'identité.

Tous s'accordaient également pour situer le vrai débat sur le terrain de la disjonction ou de l'articulation local-local et local-global, c'est-à-dire sur le terrain contesté de la connaissance/ ignorance mutuelle; de la production de la différence ; des préjugés, de l'ignorance et de la violence interethnique, comme de la communication et des identités multiples. Ce débat est récent. Il date des années 1980 . Il ne peut pas encore remplacer le débat sur la convergence, qui ne se survit dans le sens commun du grand public que grâce au cloisonnement des savoirs locaux et parce qu'il s'accorde si bien avec la visibilité à la fois locale et globale des enseignes de MacDo et de Coca-Cola. Il s'agit d'un de ces "culturescapes » imaginaires dont Appadurai explique la prolifération par la disjonction des savoirs ou des « renseignements ».

La difficulté que les uns et les autres éprouvent à déplacer le débat du terrain de la "mondialisation de la culture" aux cloisonnements et à l'effritement culturel est donc, en elle-même, un objet anthropologique qui mérite un examen attentif. On ne peut dire que les ethnologues, au moins de langue anglaise, soient passés à côté de ce débat. Au contraire, ils l'ont investi.

\section{Le champ magnétique de la "culture-com "}

La nouveauté du débat abordé par la ASA explique en partie la difficulté qu'on éprouve à déplacer le sens commun dans sa direction. Mais il me semble qu'elle n'explique pas tout. Le mot de "culture", qui, avec celui "d'identité " ou d'identification, a servi d'étendard à l'apport de l'ethnologie au débat public, a également servi de point de ralliement pour d'autres spécialistes qui sont très loin de partager le «b-a, ba " de la discipline ethnologique, à savoir l'humble enquête locale dans la durée.

Je fais référence à tous les historiens, sociologues, économistes, spécialistes de l'histoire dite culturelle, de la sociologie ou de l'économie de la "culture ». Entendez la "culture» au sens du Ministère de la culture à savoir les pratiques culturelles (musique, lecture, fréquentation des musées, spectacle vivant, etc.), l'audiovisuel, le patrimoine culturel national. Or ce domaine de compétence est très largement dominé, à juste titre, par la question de l'économie de ce domaine lucratif et créateur d'emplois, de l'impact des médias, et du contrôle (privé ou public) qui 
s'exerce ou ne s'exerce pas sur ces activités. Le fait que le mot "culture " désigne des réalités si différentes les uns des autres, bien qu'évidemment apparentées, et relève de disciplines multiples, brouille les cartes aux dépens de la clarté du message que pourraient faire passer les ethnologues.

Ce domaine, dit de la culture ou de la diversité culturelle, est l'arène d'un bras de fer entre les industries américaines d'une part, et les industries (principalement française et européenne), les gouvernements et les opinions publiques nationales qui ressentent la concurrence américaine d'autre part. Cette bataille - car bataille il y a - s'est livrée sur au moins deux terrains. Le premier est celui de l'Unesco, qui, suite à la rédaction du rapport MacBride en 1980, réclamait un "Nouvel ordre mondial de l'information et de la communication " (dit «Nomic») défavorable aux médias américains. À la suite de ce débat, les USA, la Grande Bretagne et Singapour ont quitté l'Unesco et ont cessé de cotiser jusqu'en 1997 pour la Grande Bretagne, et 2003 pour les États Unis. Le second est celui de l'Organisation mondiale du commerce qui, sous la pression des États-Unis, se déclare compétente pour soumettre les marchandises culturelles aux mêmes règles de libre échange que celles qui s'appliquent à toute autre marchandise. La France depuis la fin des années 1990 , a fait campagne pour «l'exception culturelle ". Le débat a pris de l'ampleur en se reformulant en termes de "diversité culturelle ", en faisant reconnaître celle-ci comme un droit fondamental de l'humanité, en faisant cause commune avec la protection de la biodiversité et $\mathrm{du}$ patrimoine culturel et naturel de la planète, contre les États-Unis d'Amérique qui affirment de plus en plus clairement leur unilatéralisme. L'Assemblée générale de l'Unesco doit se prononcer en octobre 2005 sur une convention relative à la diversité culturelle qui recevra sans doute le soutien de tous les pays membres, sauf les États-Unis et Israël.

L'une des difficultés à faire entendre le point de vue ethnologique dans le débat sur la non mondialisation de la culture vient donc du fait que le mot "culture" véhicule des références professionnelles, des pratiques, des traditions et des postures idéologiques ou savantes très diverses. Les industries de la culture, menacées par le géant américain, redoutent à juste titre l'américanisation des médias et la convergence des contenus "culturels» par unification des supports, dans les pays ouverts aux produits "made in USA ". Il est impossible de les faire bouger de là pour prendre en compte l'autre débat - celui du colloque ASA - qui ne dispose pas du haut parleur médiatique. Ces industries, en effet, ont un accès direct à tous les médias, de sorte que leur propre débat reçoit une énorme publicité. Deux ou trois centaines d'ethnologues ne font pas le poids contre une machine d'une telle puissance.

Cette difficulté se conjugue à un biais propre aux spécialistes de la "culture" médiatique et médiatisée. Par construction, ils sont plus sensibles à l'offre mondialisée qu'à la réception localisée des produits dits "culturels». Ils ne disposent ni du temps ni de la longue patience des ethnologues. Ils travaillent souvent à partir de données secondaires. Il est vrai qu'à considérer la masse énorme de marchandises culturelles (films, téléfilms, disques, journaux quotidiens, magazines, dépêches d'agence, photographies, livres, etc.) déversées sur le marché mondial par quelques géants comme Bertelsmann, Sony, Murdock, les majors américaines, etc., il y a lieu d'être sérieusement inquiet sur la capacité de l'humanité à digérer localement de telles masses de contenus globalisés.

Si par contre, en adoptant la posture ethnologique, on s'interroge sur la réception localisée, sur la part de la consommation culturelle de produits globalisés dans l'ensemble des pratiques des sujets, sur les tris, réappropriations, métissages et braconnages que font les usagers, alors il y a lieu, non pas tant d'être inquiet que de se poser d'autres questions, autrement plus sérieuses, que celle de la convergence des civilisations réduite aux industries dites de la « culture».

\section{L'enjeu ethnologique et le terrain}

Il existe aujourd'hui un enjeu ethnologique fort. Dans les années 1930 à 1950, il s'est agi de contrer (plus ou moins consciemment, plus ou moins adroitement) l'idéologie colonialiste et son ethnocentrisme. Il s'est agi de faire connaitre les sociétés de la tradition avant que les Philistins ne les fassent disparaître. Il s'agissait de dénoncer les ethnocides. Dans les années 1950 à 1990, il s'est agi de faire connaître et apprécier la diversité des civilisations, y compris la diversité interne de nos propres sociétés. L'outil, le levier qui a été efficacement mis en œuvre dans ce travail fut l'enquête de terrain. Rien d'autre que le terrain, mais nécessairement le terrain.

$\mathrm{Au}$ début du $\mathrm{XXI}^{\mathrm{e}}$ siècle, les ethnologues sont presque les seuls (avec quelques historiens et politologues ouverts à leurs travaux) à poser la question complexe des relations local-local et local-global à côté de la question de la convergence ou de l'américanisation, pertinente pour les industries culturelles, mais dépourvue de pertinence au regard du "cœur de métier " de l'ethnologie comme du devenir social et culturel de l'humanité. Il serait important, à mon avis, d'explorer plus avant les implications de ce débat et de s'armer pour le faire émerger sur la place publique. Si les ethnologues ne le font pas, ou si l'ethnologie s'affaiblit faute d'engagement, personne ne le fera. L'outil sera le même: le terrain. On connaît les critiques qui ont visé cet exercice canonique. Il me semble que, dans l'ensemble, elles ont été utiles et nous ont rendus plus lucides à l'égard de nous-mêmes - plus "réflexifs ". Le collectif publié sous la direction de O. Leservoisier (2005) tente de faire le bilan de ces développements. Plus que jamais, la pratique $\mathrm{du}$ terrain définit la posture rare, originale et scientifiquement efficace qui est la nôtre. 
1 Le structuralisme, comme on le sait, fonde le social sur l'échange et la communication, et la communication sur des codes mettant en œuvre des oppositions binaires. Ce que redoutait Gobineau, c'est la disparition du sens par l'abolition de la différence, du contraste pertinent. Claude Lévi-Strauss n'a pas manqué une occasion de s'élever contre le brouillage des codes et des contrastes, ainsi lorsqu'il s'est opposé à l'entrée de Marguerite Yourcenar à l'Académie française en reconnaissant que sa contribution aux lettres françaises dépassait de loin celle de bien des «Immortels » mais que l'élection d'une femme contribuerait à abolir les contrastes de genre et leur pertinence dans cette société exclusivement masculine.

2 La ASA tient un colloque annuel ordinaire, et, tous les 10 ans, un colloque extraordinaire préparé plusieurs années à l'avance, et fréquenté par un nombre considérable d'anthropologues.

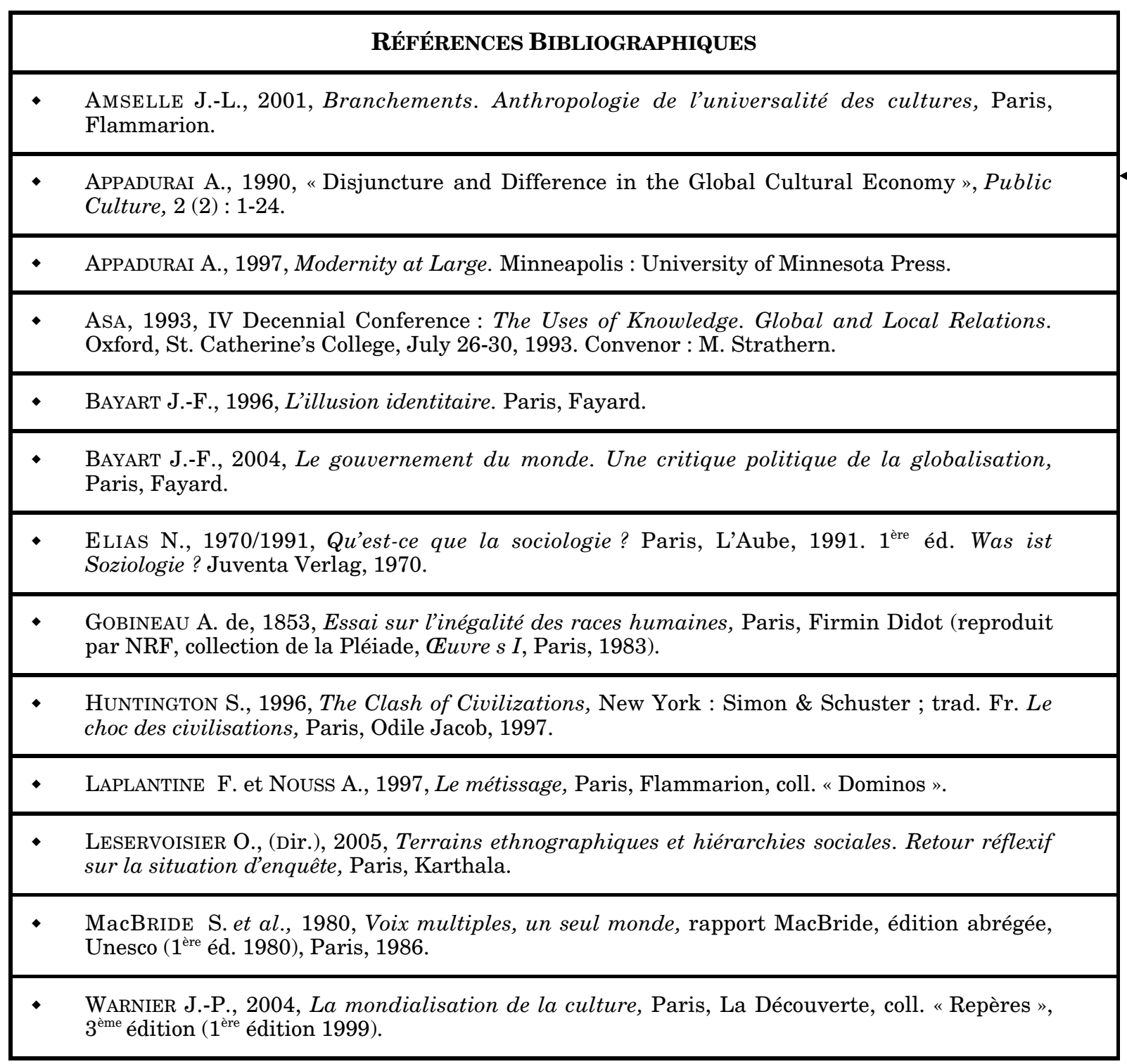

